\title{
Erratum to: Preface
}

\section{Mutsumi Takagi $\cdot$ Masashi Fujiwara \\ Takeshi Omasa $\cdot$ Sanetaka Shirahata}

Published online: 22 June 2012

(C) Springer Science+Business Media B.V. 2012

\section{Erratum to: Cytotechnology \\ DOI 10.1007/s10616-012-9460-3}

Unfortunately, the author name has been misspelled as Masamichi Fujiwara. It should read Masashi Fujiwara.

The online version of the original article can be found under doi:10.1007/s10616-012-9460-3.

M. Takagi · M. Fujiwara

Hokkaido University, Sapporo, Japan

T. Omasa

University of Tokushima, Tokushima, Japan

S. Shirahata $(\bowtie)$

Kyushu University, Fukuoka, Japan

e-mail: sirahata@grt.kyushu-u.ac.jp 